\title{
A novel method of medical image registration based on resample and wavelet transform
}

\author{
Lin Chengxin ${ }^{1, a} \quad$ Yang Yunfeng ${ }^{1, b}$ \\ ${ }^{1}$ School of Mathematics and Statistics, Northeast Petroleum University, Daqing, 163318, China \\ a1171003605@qq.com byfyang1080@126.com
}

\begin{abstract}
Key words: medical image registration; resample operator; wavelet transform
Abstract: Medical image registration is a very important technology in the field of medical image fusion, in order to meet the needs of clinical diagnosis and treatment. This paper presents a registration method of medical images based on contour features and multi-scale information. First, the resample operator is used to reduce the resolution of the couple images, and it can reduce the amount of computation effectively in the rough registration process. Second, Canny operator and method of scan line are used to extract the feature contour of the reduced dimension image. Then the principal axis method is applied to realize the rough registration based on contour lines. Finally, the subband images obtained by wavelet transform are used to accomplish the hierarchical registration. The experimental results show that the proposed method can guarantees the accuracy of registration and also reduces the amount of computation in the process of registration, and as well as in the condition of the image has a partial incomplete, we can also get the ideal registration results.
\end{abstract}

\section{Introduction}

Along with the development of science and technology, all kinds of medical instruments have been invented and put into use, and it makes the medical images have been widely used in the process of clinical diagnosis. Medical image registration is an important technique in the field of image fusion. Doctors not only can get more information about the diseases, but also can make a more detailed treatment plan by using the registration of images. The essence of registration is how to determine the registration parameters between the target image and the reference image which including translation, rotation and scaling, and so on. And at last the couple of images could completely aligning in the geometrical position. There are a lot of methods for the registration of medical images ${ }^{[1,2,3]}$.

The wavelet transform is an effect time-frequency analysis tool developed in the 1980s, and it has been applied in the image processing field widely after Mallat ${ }^{[4]}$ presented the fast decomposition algorithm.. By using wavelet transform in the procedure of images' registration, the amount of computation can be reduced effectively. Thus, the wavelet transform also has been introduced to the medical image registration. The scholars have made a lot of new research results in image registration based on wavelet transform theory ${ }^{[5,6,7,8]}$. Image registration based on the principal axes method is proposed by Alpert, the main idea is to use the contour of the image to complete registration process ${ }^{[9]}$.

In this paper, a novel method of registration of medical images is proposed, which is based on the contour line and simplified wavelet transform mainly. In addition, the resample method is selected to reduce pixels of the couple images before the registration.

\section{Resampling of an image}

The image dimension is reduced by the formula as follows:

$$
I(x, y)=f(x, y) * g(x, y)
$$

Where $f(x, y)$ denotes the original image, $I(x, y)$ denotes the sampling results and 
$g(x, y)$ denotes mask function,

According to the formula (1), an instance of mask showed in (2) is proposed which can be used to reduce the low frequency information of the image.

$$
\left(\begin{array}{ll}
1 & 1 \\
1 & 1
\end{array}\right)
$$

By using the mask, the resolution of the image can be reduced to 1/4 comparing with the original. It will reduce the calculation effectively in the procedure of the registration.

\section{Rough registration based on the principal axis}

Medical image registration based on contour feature is realized by the principal axis method. The details are as follows.

\section{A. The edge feature extraction of the medical image}

Features are often used to express an image for that features not only can reduces the operation data effectively, but also save the time and storage space in processing. In the paper, Canny edge operator is used to extract the edge features because the operator not only can obtain edge features of an image but also can overcome the affection of noise.

\section{B. Extraction of the Contour line}

On the basis of the above features, the scanning line is used to obtain the contour lines of image. In Figure.1, the specific method is to use parallel horizontal axis straight line by line to scan in horizontal direction and vertical direction and record the first and the last intersection line and the row (column) in each row. The points are bounded into a point set and a boundary point set is obtained after remove the repeating points.

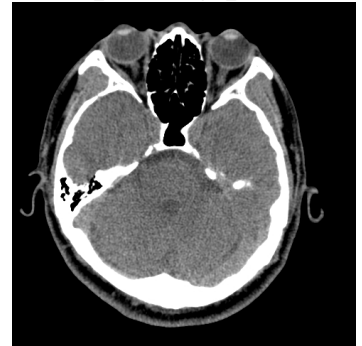

(a) Original image

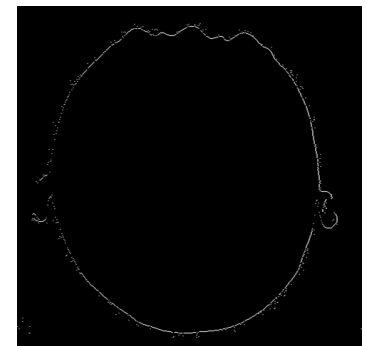

(b) Contour line

Figure.1 Contour line extraction of an image

\section{Rough registration}

By using of the Principal Axes method, the rough registration is accomplished. The detail is as follows. The inertia matrixes of the couple images are obtained by using formula (3) at first, and based on this, the long axes and the short axes of the couple images can be obtained. In the following, the angle between two long axes is calculated with formula (4), the angle between the two short axes is calculated with formula (4) too. The average value of the two angle obtained above will used to be rotation parameter in the rough registration.

$$
I=\left(\begin{array}{cc}
\sum_{i=1}^{N}\left(x_{i}-\bar{x}\right)^{2} & -\sum_{i=1}^{N}\left(x_{i}-\bar{x}\right)\left(y_{i}-\bar{y}\right) \\
-\sum_{i=1}^{N}\left(x_{i}-\bar{x}\right)\left(y_{i}-\bar{y}\right) & \sum_{i=1}^{N}\left(y_{i}-\bar{y}\right)^{2}
\end{array}\right)
$$

Where $\left(x_{i}, y_{i}\right)$ denotes pixel of the contour line, $(\bar{x}, \bar{y})$ denotes the center of the contour line.

$$
\theta=\arccos \left(\frac{\left(v_{1}, v_{2}\right)}{\left|v_{1}\right|\left|v_{2}\right|}\right)
$$

Where $\left(v_{1}, v_{2}\right)$ means the inner product of vector and $\bullet \mid$ represents for the norm of vector.

By using the centers of the couple images' contour line, the translation parameter can be 
obtained with the distance of the couple contour lines' centers.

So far the registration parameters of rough registration can be obtained.

\section{Hierarchical registration based on wavelet decomposition}

In the processes of the hierarchical registration, the couple images to be registered are decomposed by wavelet transform at first. And the processes can be divided into two parts of registration of high frequency subbands and original image. In the processes of the high frequency subbands' registration, reference images and target images are decomposed by wavelet transform with k-level. The registration process is begun from subband $H L_{k}$ to subband $H L_{k-2}$, and the initial search parameter is $\left(\Delta x / 2^{k}, \Delta y / 2^{k}, \Delta \theta\right)$. After this, the original images are selected as the registration object with initial parameter $\left(\Delta x_{0}, \Delta y_{0}, \Delta \theta_{0}\right)$, and

$$
\left(\Delta x_{0}, \Delta y_{0}, \Delta \theta_{0}\right)=2\left(\Delta x_{1}, \Delta y_{1}, \Delta \theta_{1}\right)
$$

$\left(\Delta x_{1}, \Delta y_{1}, \Delta \theta_{1}\right)$ is the registration parameter of subbans $H L_{1}$

\section{Experiments and results}

The target images and reference images are all $512 \times 512$ and the gray value is 8 bits. In the experiments, both target image and reference image are processed by the resample process, and the resolution of the resampled images are $256 \times 256$. The number of wavelet decomposition is 2 times. Figure 2 is for the registration result of CT images and Figure 3 shows the registration result between MRI images and Figure 4 show the registration results for incomplete images. Tab. 1 shows the experimental results of the method proposed in the paper.

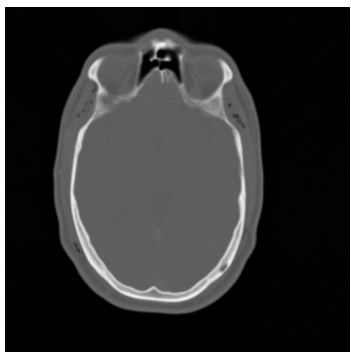

(a) Target image

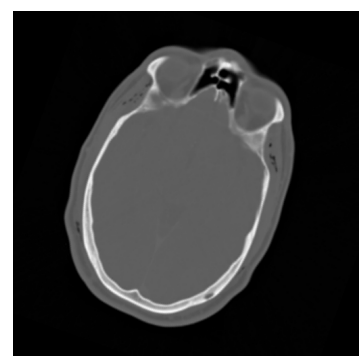

(b) Reference image

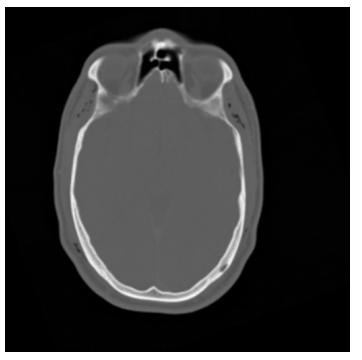

(c) Registration image

Figure $2 \mathrm{CT}$ image registration

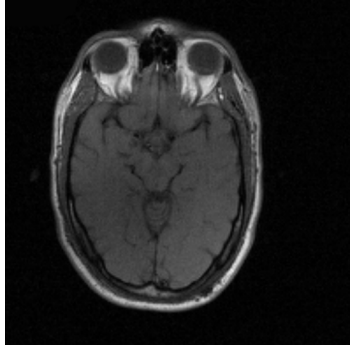

(a) Target image

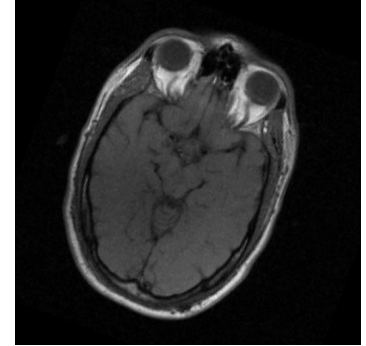

(b) Reference image

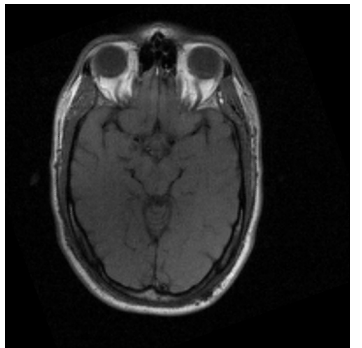

(c) Registration image

Figure 3 MRI image registration 


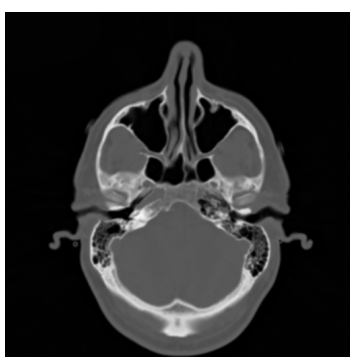

(a) Target image

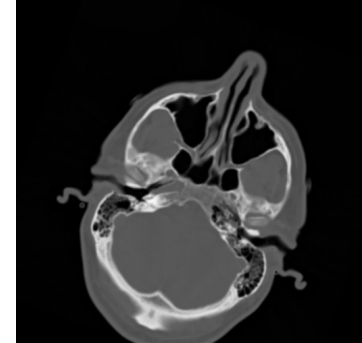

(b) Reference image

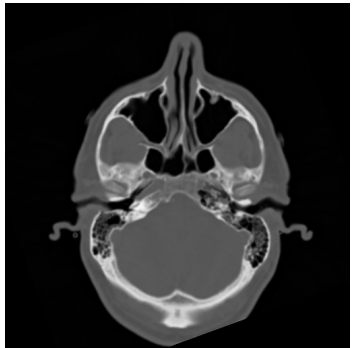

(c) Registration image

Figure 4 registration for incomplete images

Tab.1 Parameters compare with registration based on simplified multi wavelet transform

\begin{tabular}{|l|c|c|c|c|c|c|}
\hline \multirow{2}{*}{} & \multicolumn{2}{|c|}{ The registration parameters } & \multicolumn{3}{c|}{ The initial parameters } \\
\cline { 2 - 7 } & $\Delta x$ & $\Delta y$ & $\Delta \theta$ & $\Delta x$ & $\Delta y$ & $\Delta \theta$ \\
\hline Figure 2 & 19.94 & 15.03 & 19.98 & 20 & 15 & 20 \\
\hline Figure 3 & 19.98 & 14.95 & 19.98 & 20 & 15 & 20 \\
\hline Figure 4 & 19.98 & 14.99 & 20.00 & 20 & 15 & 20 \\
\hline
\end{tabular}

\section{Conclusion}

A medical image registration method based on resample and simplified wavelet transform is proposed in the paper. This method can be divided into three parts, image resampling, rough registration and hierarchical registration. the method of resample is used to reduce the computation of data in the registration process. The rough registration is used to obtain initial registration parameters. And the hierarchical registration based on simplified wavelet decomposition improves the registration accuracy. As the experimental results shown, it is a rapid and effective method for medical image registration.

\section{Reference}

[1] J. B. Antoine Maintz, Max A. Viergever, A survey of medical image registration[J], Medical Image Analysis, 1998, 2(1),:1-36

[2] Chen Xianyi, Zhou Carrier. Common methods of medical image registration and classification of [J]. Information technology, 2008(7):P17-19.

[3] Li Xiongfei, Zhang Cunli, Li Hongpeng et al. Development of medical image registration technology [J]. Computer science, 2010, 37 (7): 27 - 33.

[4] S. G. Mallat. Multifrequency channel decompositions of image and wavelet models[J]. IEEE Transaction on Acoustics, Speech and Signal Processing. 1989,37(12):2091-2110

[5] Zhang S., Tang S., Dong J. Automatic Registration of Medical Image Based on Wavelet Pyramid and Contour Feature[J]. Computer Simulation, 2008,25(5):205-209.

[6] SHARMAN R, TYLER J M, PIANYKH O S. A fast and accurate method to register medical images using Wavelet Modulus Maxima[J]. Pattern Recognition Letters, 2000(21):447-462.

[7] XU R, CHEN Y. Wavelet-based Multire solution Medical Image Registration Strategy Combining Mutual Information with Spatial Information [J]. International Journal of Innovative Computing, Information and Control, 2007, 3(2):285-296.

[8] Yunfeng Yang and Zhixun Su. A Novel Medical Image Registration Algorithm Based on Contour Features and Multi wavelet Transform, Journal of Information \& Computational Science, 2012, 9(6):1413-1420.

[9] ALPERT N M, BRADSHAW J F, KENNEDY D, et al. The Principal Axes Transformation-A Method for Image Registration [J]. The Journal of Nuclear Medicine, 1990, 31(10):1717-1722. 